\title{
Gradhiva
}

\section{Franck Beuvier. Danser les funérailles. Associations et lieux de pouvoir au Cameroun}

Paris, Éditions de l'EHESS, coll. « En temps \& lieux », 2014

\section{Jean-François Gossiaux}

\section{(2) OpenEdition}

\section{Journals}

Édition électronique

URL : http://journals.openedition.org/gradhiva/3302

DOI : $10.4000 /$ gradhiva.3302

ISSN : 1760-849X

\section{Éditeur}

Musée du quai Branly Jacques Chirac

Édition imprimée

Date de publication : 7 décembre 2016

Pagination : 244-245

ISBN : 978-2-35744-094-4

ISSN : 0764-8928

\section{Référence électronique}

Jean-François Gossiaux, « Franck Beuvier. Danser les funérailles. Associations et lieux de pouvoir au Cameroun », Gradhiva [En ligne], 24 | 2016, mis en ligne le 07 décembre 2016, consulté le 23 septembre 2020. URL : http://journals.openedition.org/gradhiva/3302 ; DOI : https://doi.org/10.4000/gradhiva. 3302

Ce document a été généré automatiquement le 23 septembre 2020

(c) musée du quai Branly 


\section{Franck Beuvier. Danser les funérailles.} Associations et lieux de pouvoir au

\section{Cameroun}

Paris, Éditions de l'EHESS, coll. « En temps \& lieux », 2014

Jean-François Gossiaux

\section{RÉFÉRENCE}

Franck Beuvier. Danser les funérailles. Associations et lieux de pouvoir au Cameroun. Paris, Éditions de l'EHESS, coll. «En temps \& lieux », 2014, 304 p. 


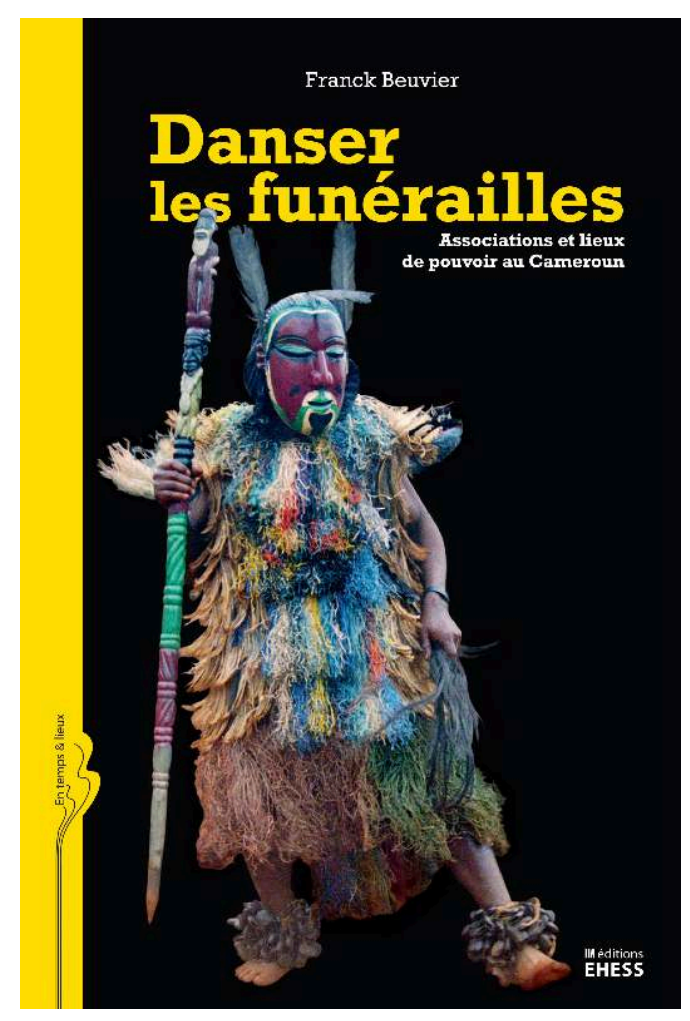

1 L'étonnement est le premier instrument de travail de l'anthropologue, sans lequel celui-ci se retrouve à enfiler des perles rhétoriques. Logiquement, donc, et paradoxalement, plus l'anthropologue travaille, mieux il connait son terrain, et plus il risque de se trouver heuristiquement démuni. Il lui arrive cependant d'être sauvé par ce que l'on pourrait appeler des "surprises à la puissance deux », c'est-à-dire des faits qui viennent à rebours des connaissances qu'il s'est forgées avec ses premiers étonnements. C'est ce genre de surprise qui a radicalement orienté, sinon déclenché, la recherche entreprise par Franck Beuvier en pays bamiléké, et qui nous vaut ce livre important - et étonnant. Assistant à une cérémonie de funérailles, l'ethnographe voit son savoir perturbé par le spectacle de jeunes roturiers (les spécialistes du Cameroun parlent de "cadets sociaux») arborant, à la satisfaction générale, des attributs qui en toute logique (ethnographique) devraient leur être interdits. Il s'ensuit une chaîne de remises en question des concepts tenus pour acquis: tradition ("coutume»), notabilité, légitimité... Au bout du compte et de la recherche, une logique est retrouvée, qui est historiquement articulée autour des funérailles, théâtre privilégié du jeu social bamiléké, avec les associations de danse dans les rôles principaux.

2 La démonstration se déroule en dix chapitres, dont chacun constitue en lui-même une leçon d'ethnographie. Il est d'abord question des morts, de leur relation aux vivants $-i$. $e$. de la relation des vivants aux morts - et de la pression qu'ils exercent sur la société et sa hiérarchie. Les funérailles sont à cet égard le moment - et le lieu - crucial. Celles-ci, en l'occurrence, ne sont pas une cérémonie d'enterrement, mais une célébration organisée - ou non, mais en tout état de cause toujours " réclamée » par le défunt - un certain temps, parfois long, après le décès. Les enjeux proprement sociaux s'y mêlent aux rapports problématiques entretenus avec le disparu, et ce d'autant plus que la condition notabiliaire du célébrant est élevée. Dans ce contexte où se conjuguent obligations rituelles et exigences de prestige, les performances des associations de danse occupent une place centrale. Ces dernières se situent dans la filiation des 
collectivités masculines, des " fraternités », telles qu'elles existaient jusqu'au milieu du $\mathrm{xx}^{\mathrm{e}}$ siècle. L'ouvrage s'attache aux valeurs et au fonctionnement de ces anciennes associations, et analyse leur évolution au sein de la société coloniale puis au moment de l'indépendance et de la mise en place d'une politique culturelle à l'origine, d'une certaine manière, des groupes qui animent aujourd'hui les funérailles. Leurs performances se déclinent en véritables "genres", dont l'auteur découvre les conditions secrètes d'élaboration. Le chapitre final fait ressortir la dimension coutumière de ces groupes et leur place dans la relation rituelle aux morts.

3 L'ouvrage emporte la conviction par la finesse de ses analyses et la rigueur logique de ses enchaînements autant que par la précision et la richesse de son ethnographie. La difficulté d'écriture inhérente à la description méticuleuse de situations et de séquences rituelles n'est pas ici exclusive d'un réel plaisir de lecture. Nous retiendrons particulièrement l'hallucinant jeu avec le feu du groupe Kana (chapitre VII). La cocasserie n'est pas absente, par exemple dans cette scène de chaises musicales à laquelle donne lieu - «lutte des places » stricto sensu - le placement des notables lors des funérailles (chapitre II). Mais si la drôlerie peut émerger de certaines situations, le ton n'abuse jamais de l'humour ni ne donne à aucun moment dans le pittoresque ou l'ironie.

4 L'intérêt du lecteur est de surcroît stimulé par la sorte d'inconfort dans lequel il se trouve placé. Il ne s'agit pas, en l'occurrence, du malaise suscité par la description de quelque spectacle impressionnant ou dérangeant mais de l'impression constante d'être dans le paradoxe - à l'image du paradoxe à l'origine du livre, de cette incongruité rituelle où les acteurs ne sont pas ceux qui devraient l'être (ou ceux qu'ils devraient être). Le titre, déjà, accroche l'attention par l'alliance insolite de la danse et des funérailles. Puis c'est le concept même de rituel qui se trouve quelque peu chahuté à la lecture. Même s'il est vrai que, contrairement aux idées reçues, les rites se répètent rarement à l'identique et que leur déroulement n'est pas exempt d'enjeux, ceux qui nous sont donnés à voir ici poussent au maximum le principe d'incertitude en systématisant les variations et en fabriquant de l'imprévu par la mise en compétition des acteurs.

5 Surtout, le lecteur éprouve la sensation (inconfortable et stimulante, donc) d'être plongé en permanence et simultanément dans deux mondes contradictoires: d'une part, il reconnaît les réalités familières du monde "moderne ", de l'autre, il se trouve immergé dans des réalités inverses, celles du monde de la "coutume», de la «tradition ». On est loin, pourtant, des classiques variations sur le thème " tradition et modernité », et l'on échappe aux analyses routinières de la tension entre ces deux pôles. Les réalités contradictoires sont beaucoup plus profondes et profondément imbriquées - jusqu'à ne pas être contradictoires.

6 La société coutumière se révèle ainsi totalement monétarisée, à l'image, et au-delà, des sociétés de l'hypermodernité. Tout s'achète, tout se paie. On achète des positions, des droits, des choses pour les défunts... La vie sociale a des allures d'immense potlatch. Peut-on parler de commerce? Serait-on dans le paradigme du don? Il y a là un matériau de choix pour l'anthropologie économique. Et, bien sûr, du pain bénit pour les tenants de la psychanalyse (le paiement, la dette...).

7 On paie notamment pour accéder aux associations. Associations : le mot-clé du livre, au cœur de la démonstration - le pont entre les deux mondes. Le mot ressortit à la «modernité » et au monde du contrat - il participe même de leur définition. Mais il 
appartient aussi au monde de la coutume. Des concepts comme ceux de société (" secrète »), de confrérie, de fraternité, de réunion, sans compter ceux qui relèvent, hors traduction, des catégories indigènes, sont subsumables (et subsumés ici) sous celui d'association. Le terme a été mis en avant par Robert Harry Lowie dans Primitive Society (1920), accompagné d'exemples tirés notamment de l'ethnographie amérindienne ${ }^{1}$. Il désigne «toutes les unités sociales qui ne sont pas fondées sur la parenté comme facteur déterminant ». Si l'usage en a été peu développé en anthropologie, pour se voir essentiellement cantonné au domaine de la sociologie («nos» sociétés modernes), il reste de la proposition de Lowie que le phénomène associatif peut être considéré comme universel, dans le sens où rien ne permet de le limiter à tel type de société, et notamment pas d'en faire un marqueur de la frontière entre « nous » et les « autres ».

8 C'est ainsi qu'il fait le lien entre les sociétés « coutumière » et « contemporaine » (c'està-dire ressortissant à "notre " monde globalisé) au Cameroun. Les guillemets, en l'occurrence, s'imposent plus encore que d'ordinaire. Car il s'agit en fait du même monde : il n'y a qu'une seule société bamiléké, qui est le sujet unique du livre. La même réalité est à l'œuvre, à travers des modalités différentes, changeantes. Et c'est précisément parce qu'il s'agit toujours de la même réalité que le changement est possible. « Plus ça change, plus c'est la même chose » ou plutôt, ici, « plus c'est la même chose, plus ça change». L'histoire et l'ethnographie mobilisées par Beuvier le démontrent: c'est à travers la pérennité de la coutume que les cadets sociaux sont parvenus au pouvoir.

9 Cette pérennité a pris concrètement la forme d'une continuité structurelle et historique entre les différents "types" d'associations à fonctions coutumières, en l'occurrence et pour ce qui nous intéresse les associations de danse - anciennes ou contemporaines, "traditionnelles» ou "modernes ». Après que le Cameroun eut obtenu son indépendance, son premier président, Ahmadou Ahidjo, s'efforça de mettre en œuvre une politique culturelle censée promouvoir, dans le pays comme vis-à-vis de l'étranger, l'unité et la modernité de la nation en valorisant la richesse de ses arts traditionnels, au premier rang desquels la danse. Dans ce contexte, les anciennes associations, les confréries, furent supplantées par des associations " culturelles » plus à même de réaliser ce programme et qui en reprirent les fonctions coutumières, sans solution de continuité. Les cadets, qui avaient joué un rôle essentiel dans ce moment d'institutionnalisation, acquirent ainsi leur dimension d'acteurs rituels, en même temps qu'ils accédaient, plus généralement, au pouvoir.

Outre son ethnographie rigoureuse et brillante, sa mise en contexte historique précise et pertinente, l'ouvrage de Beuvier tire sa puissance démonstrative de cette sorte de coup d'audace théorique : ignorer la frontière sémantique entre tradition et modernité, et rendre opératoire le concept d'association en reprenant (au sérieux) son acception anthropologique. C'est la ré-universalisation de ce concept qui a permis d'unifier la problématique et de résoudre les contradictions liées aux clivages fantasmés des sciences sociales. Au-delà de sa dimension africaniste, la démonstration constitue une véritable leçon d'anthropologie. 


\section{NOTES}

1. Voir Michel Izard, "Association", in Pierre Bonte et Michel Izard (dir.), Dictionnaire de l'ethnologie et de l'anthropologie, Paris, PUF, 2000 : 94-97.

\section{AUTEURS}

\section{JEAN-FRANÇOIS GOSSIAUX}

gossiaux@msh-paris.fr 